\title{
IMPROVEMENT OF INTRAOPERATIVE ANTIBIOTIC Prophylaxis in Prolonged Cardiac Surgery by Automated Alerts in the Operating Room
}

\author{
Giorgio Zanetti, MD, MS; Hugh L. Flanagan, Jr., MD; Lawrence H. Cohn, MD; Richard Giardina, RN, MPH, CIC;
} Richard Platt, MD, MS

\begin{abstract}
OBJECTIVE: To assess the impact of an automated intraoperative alert to redose prophylactic antibiotics in prolonged cardiac operations.

DESIGN: Randomized, controlled, evaluator-blinded trial.

SETTING: University-affiliated hospital.

PATIENTS: Patients undergoing cardiac surgery that lasted more than 4 hours after the preoperative administration of cefazolin, unless they were receiving therapeutic antibiotics at the time of surgery.

INTERVENTION: Randomization to an audible and visual reminder on the operating room computer console at 225 minutes after the administration of preoperative antibiotics (reminder group, $n=137)$ or control $(n=136)$. After another 30 minutes, the circulating nurse was required to indicate whether a follow-up dose of antibiotics had been administered.

RESULTS: Intraoperative redosing was significantly more frequent in the reminder group (93 of 137;68\%) than in the control group (55 of 136; 40\%) (adjusted odds ratio, 3.31; 95\% confidence interval, 1.97 to $5.56 ; P<.0001$ ). The impact of the reminder was even greater when compared with the 6 months preceding the study period (129 of $480 ; 27 \% ; P<.001)$, suggesting some spillover effect on the control group. Redosing was formally declined for 19 of the 44 patients in the reminder group without redosing. The rate of surgical-site infection in the reminder group $(5$ of $137 ; 4 \%)$ was similar to that in the control group ( 8 of $136 ; 6 \% ; P=.42$ ), but significantly lower than that in the pre-study period ( 48 of $480 ; 10 \% ; P=.02$ ).

CONCLUSION: The use of an automatic reminder system in the operating room improved compliance with guidelines on perioperative antibiotic prophylaxis (Infect Control Hosp Epidemiol $2003 ; 24: 13-16$ ).
\end{abstract}

Perioperative antibiotic prophylaxis is among the most effective methods to reduce the incidence of surgicalsite infections for many types of surgical procedures. ${ }^{1}$ However, its efficacy has been shown to diminish during long procedures. ${ }^{2-4}$ This diminished efficacy has prompted recommendations to redose the antibiotic during procedures of prolonged duration. ${ }^{1,5-8}$ Despite these recommendations, intraoperative redosing is often omitted. ${ }^{9}$

We therefore developed an audible alarm and message delivered via computer consoles in the operating rooms to remind surgical teams to consider intraoperative redosing of antibiotics at the time such antibiotics appeared to be indicated. The purpose of this randomized, controlled study was to quantify the impact of this reminder system on redosing of antibiotics during prolonged cardiac procedures.

\section{METHODS}

Patients undergoing operations between March 23 and June 23, 2000, in the Division of Cardiac Surgery at Brigham and Women's Hospital, Boston, Massachusetts, were evaluated for inclusion in this prospective, randomized, controlled study. The study was approved by the institutional review board.

All patients were allocated to either activation of an automated reminder system or no intervention (control), based on a case number assigned to every surgical procedure performed in the hospital, independent of the study itself. All operating rooms contained computers linked to the hospital's central information system. These computers are used during procedures by the circulating nurse to both obtain and record information, including the start time of procedures.

The reminder system consisted of an audible alarm generated by the operating room computer for patients whose procedure lasted more than 225 minutes after the administration of a first preoperative dose of antibiotic. This time of activation was chosen because the guidelines in force in the hospital recommended cefazolin as prophylaxis for cardiac surgery and suggested intraoperative redosing intervals of 240 minutes for this antibiotic.

Drs. Zanetti and Platt and Mr. Giardina are from Channing Laboratory, Department of Medicine, Brigham and Women's Hospital, Harvard Medical School, and the CDC Eastern Massachusetts Prevention Epicenter, Boston, Massachusetts. Dr. Zanetti is also from the Division of Infectious Diseases, Department of Internal Medicine, University Hospital, Lausanne, Switzerland. Dr. Flanagan is from the Department of Anesthesia and Dr. Cohn is from the Division of Cardiac Surgery, Department of Surgery, Brigham and Women's Hospital, Harvard Medical School, Boston, Massachusetts. Dr. Platt is also from the Department of Ambulatory Care and Prevention, Harvard Medical School, and Harvard Pilgrim Health Care, Boston, Massachusetts; and the HMO Research Network Center for Education and Research in Therapeutics.

Address reprint requests to Giorgio Zanetti, MD, MS, Division of Infectious Diseases, University Hospital, 1011 Lausanne, Switzerland.

Supported in part by a Centers for Disease Control and Prevention cooperative agreement, UR8/CCU115079, supporting the CDC Eastern Massachusetts Prevention Epicenter. Dr. Zanetti is supported by grants from the University Hospital of Lausanne and the Leenaards Foundation, Lausanne, Switzerland. 


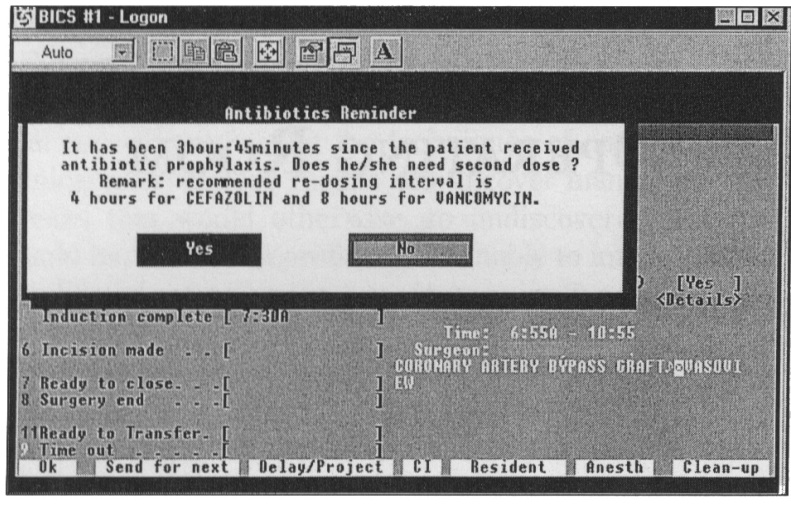

FIGURE 1. Message displayed on the console of the operating room computer together with an audible alarm.

Simultaneous with the alarm, a message was displayed on the same computer console. This message cited hospital guidelines on intraoperative redosing of antibiotics, and asked whether the surgical team was considering redosing (a facsimile is shown in Fig. 1). A reply was required to clear the display for any other use. If the response indicated that redosing of prophylaxis was planned, a new audible alert and screen appeared 30 minutes later, asking whether redosing had actually been performed. We did not attempt to document the circulation of the information provided by the reminder system within the surgical team.

For each eligible operation, the following data were manually abstracted from the anesthesia record: age and gender of the patient, the American Society of Anesthesiologists score, the date and type of surgery, the surgeon, the time of all antibiotic administrations, and the times of skin incision and closure. The duration of surgical procedures was calculated as the time elapsed between administration of a first, preoperative dose of an antibiotic and skin closure. Operations were categorized as including or not including coronary artery bypass graft to avoid comparisons between small subgroups. The same data were collected during the 6 months preceding the study to estimate the magnitude of a possible overall change in the practice of intraoperative redosing due to awareness of the ongoing study.

Procedures were excluded from analyses if they were shorter than 240 minutes, if no preoperative antibiotic prophylaxis was recorded, if information on the duration of surgery was unavailable, if antibiotics other than cefazolin were used for prophylaxis, or if therapeutic antibiotics were being given at the time of surgery.

The primary outcome was the proportion of patients who had been appropriately given an intraoperative redose among those whose surgery lasted at least 240 minutes after a first, preoperative dose of cefazolin. Intraoperative redosing was defined as the administration of a second dose of cefazolin at any time before surgical closure, as noted in the anesthesiologist's records.

A secondary outcome was the occurrence of surgical-site infection. Infection surveillance was performed prospectively by an experienced infection control practi-

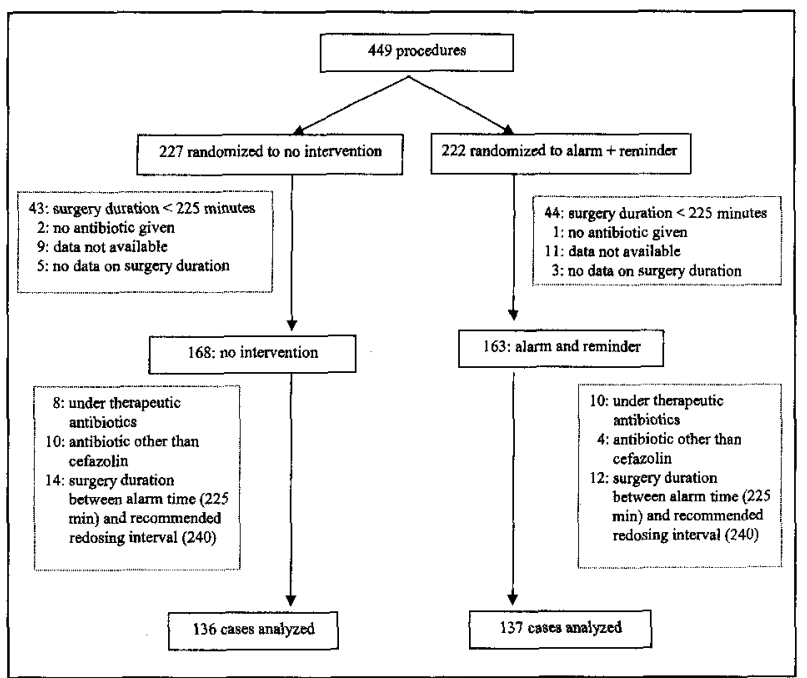

FIGURE 2. Assignment of patients to reminders and reasons for exclusion from final analysis.

tioner who was unaware of the patients' status regarding the redosing reminder. The surveillance method used the National Nosocomial Infections Surveillance System criteria ${ }^{1}$ and included both inpatient components and postdischarge information from the surgeons' office.

To compare the patients who were exposed to the reminder system with those who were not, we used the two-sided Wilcoxon rank sum test for continuous variables and the chi-square test for proportions. The significance level was .05 in all tests. Significant univariate predictors of intraoperative redosing were then candidates for inclusion in a logistic regression model that was built through a backward selection process. The absence or presence of exposure to the reminder was always forced in the model. The model was then tested for confounding by each of the excluded covariates. The Wald test was used to report the significance level of the predictors in the final model.

The odds of surgical-site infection were compared using the same strategy. Their trend was also assessed across the pre-study period, the control group, and the reminder group, with the likelihood ratio test used to assess deviance from linearity.

Statistical analyses were performed with STATA 6.0 statistical software (STATA Corp., College Station, TX).

\section{RESULTS}

Four hundred forty-nine cardiac operations were performed during the study period (Fig. 2) and allocated to either the intervention (227 patients) or the control (222 patients) group. Of them, 331 procedures in 331 patients (168 in the intervention group and 163 in the control group) reached the point where the reminder system could be activated. Two hundred seventy-three procedures (137 in the intervention group and 136 in the control group) could eventually be documented as eligible for intraoperative redosing of cefazolin according to the guidelines, and were therefore included in the primary analysis of efficacy 
TABLE 1

Characteristics of THE Patients*

\begin{tabular}{|c|c|c|c|}
\hline \multirow{2}{*}{$\frac{\text { Characteristlc }}{\text { Mean age, y (range) }}$} & $\begin{array}{c}\text { No } \\
\text { Intervention } \\
(n=136)\end{array}$ & \multicolumn{2}{|c|}{$\begin{array}{l}\text { Alarm Plus } \\
\text { Reminder } \\
(n=137)\end{array}$} \\
\hline & 67 (33 to 92$)$ & 65 & $(29$ to 93$)$ \\
\hline Male & $83(61 \%)$ & 96 & $(70 \%)$ \\
\hline \multicolumn{4}{|l|}{ ASA score } \\
\hline 2 & $6(4 \%)$ & 7 & $(5 \%)$ \\
\hline 3 & $101(74 \%)$ & 112 & $(82 \%)$ \\
\hline 4 & $22 \quad(16 \%)$ & 12 & $(9 \%)$ \\
\hline Unknown & $7 \quad(5 \%)$ & 6 & $(4 \%)$ \\
\hline \multicolumn{4}{|l|}{ Type of surgery } \\
\hline CABG & $50 \quad(37 \%)$ & 46 & $(34 \%)$ \\
\hline $\begin{array}{l}\text { Other cardiac } \\
\text { procedures }\end{array}$ & $86 \quad(63 \%)$ & 91 & $(66 \%)$ \\
\hline $\begin{array}{l}\text { Mean duration of } \\
\text { procedure, min (range) }\end{array}$ & 354 (241 to 737$)$ & 352 & ( 242 to 637$)$ \\
\hline
\end{tabular}

ASA = American Society of Anesthesiologists; CABG = coronary artery bypass graft.

*There was no significant difference between the two groups.

of the reminder system. Patients' characteristics were similar in the two groups (Table 1). The distribution of surgeons was also homogeneous between the two groups (data not shown).

In the control group, $55(40 \%)$ of 136 patients received intraoperative redosing (Fig. 3 ). This proportion was significantly higher in patients randomized to the automatic reminder system (93 of $137 ; 68 \% ; P<.001$ ). In this group, the administration of a second dose of antibiotic was formally declined in $19(14 \%)$ of 137 cases. It was noted to have been administered in the electronic system in an additional 19 (14\%) of the cases, but this was not reported on the anesthesia record. For purposes of analysis, we classified these 19 cases as having received no redosing. Of note, the proportion of patients who received a redose in the control group (40\%) was significantly higher than the comparable proportion during the 6 months before the intervention started (129 of $480 ; 27 \%$ ) (Fig. 3). This increase in the control group was consistent with the increased awareness of the importance of redosing that resulted from all surgical personnel receiving reminders about redosing during half of their cases.

Besides exposure to the automatic reminder system, the only significant predictor of intraoperative redosing of the prophylactic antibiotic was the duration of surgery. The impact of the reminder system remained significant after adjustment to surgery duration in the multivariate analysis (Table 2).

The attack rate of surgical-site infection after procedures eligible for intraoperative redosing was 48 (10\%) of 480 during the 6 months preceding the study period. During the study period, it declined to $8(6 \%)$ of 136 in the control group and to 5 (4\%) of 137 in the intervention group ( $P=.4$ compared with the control group, and $P=.02 \mathrm{com}-$

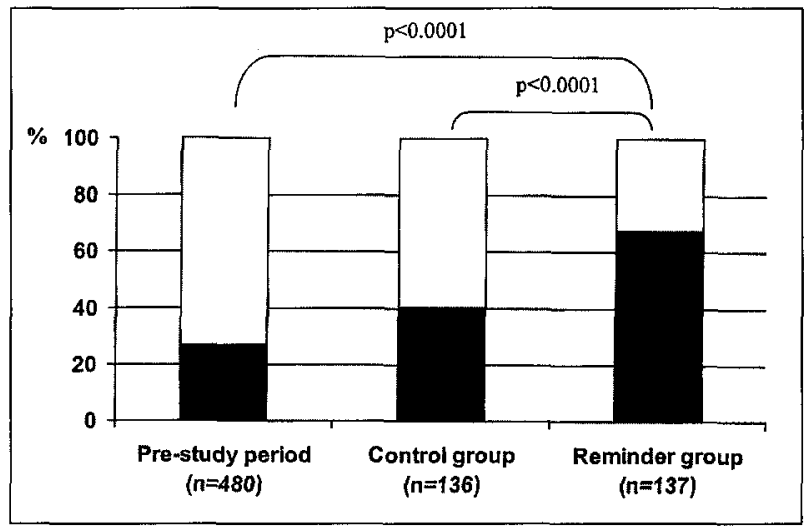

FIGURE 3. Proportion of patients who received an intraoperative redose of a prophylactic antibiotic.

TABLE 2

Independent Predictors of Intraoperative Redosing of Prophylactic Antibiotics During Cardiac Surgery*

\begin{tabular}{|c|c|c|c|}
\hline Predictor & $\begin{array}{l}\text { Ad]usted Odds } \\
\text { Ratlo for } \\
\text { Intraoperatlve } \\
\text { Redosing }\end{array}$ & $\mathrm{Cl}_{95}$ & $\boldsymbol{p t}$ \\
\hline Alarm + reminder & 3.31 & 1.97 to 5.56 & $<.0001$ \\
\hline Duration of surgery ${ }^{\ddagger}$ & 1.62 & 1.30 to 2.03 & $<.0001$ \\
\hline \multicolumn{4}{|c|}{$\begin{array}{l}\mathrm{Cl}_{95}=95 \% \text { confidence interval. } \\
\text { "Characteristics that were considered as potential confounders but not retained in the final } \\
\text { model included age and gender of the patient, the American Society of Anesthesiology score, } \\
\text { the date and type of surgery, and the surgeon. } \\
\text { tWald test. } \\
\text { FTime elapsed between administration of preoperative antibiotics and skin closure. Odds ratio } \\
\text { for every additional hour of surgery. }\end{array}$} \\
\hline
\end{tabular}

pared with the pre-study period). The infection rate in the intervention group remained significantly lower than that observed during the pre-study period after adjustment for the type of surgery, another independent predictor. Moreover, if we consider the control group to have received an intermediate intervention because of the heightened awareness about redosing during the randomized trial, there was a significant trend, with the highest rate of surgical-site infection among procedures before the study period and the lowest in the group exposed to the reminder system $(P=.01)$.

\section{DISCUSSION}

The use of this automatic reminder system was associated with a significant increase in the rate of appropriate intraoperative redosing of prophylactic antibiotics from $40 \%$ to at least $68 \%$. This is almost certainly a conservative estimate of the effect of the reminder, because the same surgical teams cared for in the two groups. Therefore, all of them were aware of the intervention and may have been generally more attentive to redosing. The fact that redosing occurred more frequently in the control group than during similar procedures in the 6 months immediately preceding 
the study (27\%) supports this inference. Also, it is likely that some of the additional $14 \%$ of patients in the intervention group who had indication of redosing in their automated records but not in their separate anesthesia records actually received a redose. However, data are not available on the reliability of the anesthesia records to estimate this possible misclassification. In addition, underreporting of redosing in anesthesia records may also have occurred in the control group to a similar or even greater extent.

In a previous study, ${ }^{9}$ we found that redosing of intraoperative cefazolin was associated with a $19 \%$ reduction in the risk for surgical-site infection after operations lasting more than 4 hours. The size of the current study was too small to confirm this observation. However, we did observe a significant reduction of the risk of surgical-site infection in the reminder group compared with the pre-study period. The trend toward a lower infection rate correlates with the rate of intraoperative redosing observed in the pre-study, control, and reminder groups, respectively. We consider this result to support the practice of intraoperative redosing in cardiac surgery.

No important harmful effects resulted from the use of the reminder system. Inappropriate activation of the system occurred in only $4(1 \%)$ of 449 procedures, apparently resulting in unnecessary intraoperative redosing in one case. We requested comments when the reminders were introduced, and received no complaints suggesting that the reminder system disturbed the surgical teams. However, we did not formally evaluate their acceptance of the reminder system.

As noted earlier, the redosing of antibiotics could not be assessed in $14 \%$ of the cases because of contradictory data between the information system and the anesthesia report. In the best-case scenario where this contradictory information would actually correspond to intraoperative redosing, the reminder system would be related to an $82 \%$ (112 of 137) compliance rate for redosing.

Redosing was explicitly refused in $14 \%$ of the cases. These refusals suggest that a reminder system does not obviate the need for an education effort. They may also underscore the need for better understanding of the way people interact within the surgical team, to increase the impact of the reminder system.

There are several arguments for adopting a real-time reminder system in the operating room. It can easily be integrated into the highly structured environment that is characteristic of surgery. In addition, its implementation is inexpensive and easy if there is an existing computerized operating room management system.

Failure to give an intraoperative redose of prophylactic antibiotics in prolonged procedures is an example of a medical error. In the aggregate, these errors have been recognized to negatively impact clinical outcomes and to impose a large burden on healthcare resources. ${ }^{10}$ An automatic reminder is a useful step to reduce the risk of this error.

\section{REFERENCES}

1. Mangram AJ, Horan TC, Pearson ML, Silver LC, Jarvis WR, the Hospital Infection Control Practices Advisory Committee. Guideline for prevention of surgical site infection, 1999. Infect Control Hosp Epidemiol 1999;20:250-278.

2. Kaiser AB, Herrington JL, Jacobs JK, Mulherin JL, Roach AC, Sawyers $\mathrm{JL}$. Cefoxitin versus erythromycin, neomycin, and cefazolin in colorectal operations: importance of the duration of the surgical procedure. Ann Surg 1983;198:525-530.

3. Shapiro M, Munoz A, Tager IB, Schoenbaum SC, Polk BF. Risk factors for infection at the operative site after abdominal or vaginal hysterectomy. $N$ Engl J Med 1982;307:1661-1666.

4. Coppa GP, Eng K. Factors involved in antibiotic selection in elective colon and rectal surgery. Surgery 1988;104:853-858.

5. Dellinger EP, Gross PA, Barrett TL, et al. Quality standard for antimicrobial prophylaxis in surgical procedures. Clin Infect Dis 1994;18:422427.

6. Page CP, Bohnen JM, Fletcher JR, McManus AT, Solomkin JS, Wittmann DH. Antimicrobial prophylaxis for surgical wounds: guidelines for clinical care. Arch Surg 1993;128:79-88.

7. ASHP Commission on Therapeutics. ASHP therapeutic guidelines on antimicrobial prophylaxis in surgery. Clinical Pharmacy 1992;11:483513.

8. Martin C, the French Study Group on Antimicrobial Prophylaxis in Surgery, the French Society of Anesthesia and Intensive Care. Antimicrobial prophylaxis in surgery: general concepts and clinical guidelines. Infect Control Hosp Epidemiol 1994;15:463-471.

9. Zanetti G, Giardina RG, Platt R. Intraoperative redosing of cefazolin and the risk for surgical site infection in cardiac surgery. Emerg Infect Dis 2001;7:828-831.

10. Kohn LT, Corrigan JM, Donaldson MS, eds. To Err Is Human: Building a Safer Health System. Washington, DC: National Academy of Sciences; 1999. Available at www4.nationalacademies.org/news.nsf. 